\title{
Evaluation of Helicobacter pylori stool antigen test in comparison with conventional methods in detection of helicobacter pylori infection in dyspepsia patients
}

\author{
Sai Priya B. ${ }^{1}$, Jagadevi², Suresh K. ${ }^{3}$, M.G. Prakash ${ }^{4}$ \\ ${ }^{1}$ Dr. Sai Priya B, Assistant Professor, Department of Microbiology, Basveshwara Medical College \& Hospital, \\ Chitradurga, India, ${ }^{2}$ Dr. Jagadevi, Assistant Professor, Department of Microbiology Basveshwara Medical College \& \\ Hospital, Chitradurga, India, ${ }^{3}$ Dr. Suresh K, Professor, Department of Microbiology Jagadguru Jayadeva Murugarajendra \\ Medical College, Davangere, ${ }^{4}$ Dr. M.G.Prakash Professor, Department of General Surgery, Jagadguru Jayadeva \\ Murugarajendra Medical College, Davangere, India.
}

Corresponding Author: Dr. Jagadevi, Assistant Professor, Department of Microbiology, Basveshwara Medical College \& Hospital, SJM Campus, NH-4 Bypass Chitradurga, Karnataka, India. Email: drjagadevi@gmail.com

\begin{abstract}
Background: Helicobacter pylori colonize the human stomach and is associated with gastritis, gastric and duodenal ulcers, gastric adenocarcinoma and mucosa associated lymphoid tissue lymphoma. Owing to its momentous pathogenic role, the diagnosis of $\mathrm{H}$ pylori infection remains a subject of interest. Helicobacter can be detected by invasive and non invasive methods. Aim: The purpose of this study was to evaluate the accuracy of stool antigen test as a non invasive method for the diagnosis of $\mathrm{H}$ pylori infection. Materials and Method: A prospective study was conducted in a tertiary medical college hospital. Patients presenting with dyspeptic symptoms were subjected to endoscopy and investigated for $\mathrm{H}$ pylori infection through rapid urease test, histopathology and culture. Stool samples were also collected from these patients and tested for H pylori infection by a rapid Hpylori Stool Antigen test. H pylori status was defined as positive when both rapid urease test (RUT) and histopathology were positive or culture alone was positive for biopsy based invasive methods. Similarly a positive rapid HpSA test also indicates H pylori infection. Results: Of the 100 patients tested, 63 were $\mathrm{H}$ pylori infected by the endoscopy based invasive methods, and 54 by the non-invasive rapid HpSA test. The sensitivity, specificity and accuracy of $\mathrm{H}$ pylori stool antigen test were $85.7 \%, 88 \%$ and $91 \%$ respectively. Conclusion: The rapid HpSA test could be used as a noninvasive diagnostictest for $\mathrm{H}$ pylori infection.
\end{abstract}

Keywords: H pylori infection, Helicobacter pylori stool antigen test (HpSA), Rapid urease test (RUT).

\section{Introduction}

Helicobacter pylori is a microaerophilic gram negative, spiral shaped bacterium that colonizes the human stomach. Infection with Helicobacter pylori is associated with gastritis, gastric and duodenal ulcers, gastric adenocarcinoma and mucosa associated lymphoid tissue (MALT) lymphoma and it has been declared as a grade one carcinogen by the WHO [1].

The prevalence of H.pylori infection is $70 \%-90 \%$ in developing countries and $25 \%-50 \%$ in developed countries [2]. Person- to- person spread is the most probable mode of transmission. Faecal-oral and oraloral transmissions have been reported [3]. Chronic $\mathrm{H}$ pylori infections of the stomach are increasingly recognized as a major risk factor for the development of

Manuscript received: $8^{\text {th }}$ November 2018

Reviewed: $18^{\text {th }}$ November 2018

Author Corrected: $24^{\text {th }}$ November 2018

Accepted for Publication: $29^{\text {th }}$ November 2018 gastroduodenal disease. Eradication of H.pylori is known to alter the course of diseases caused by it and hence its rapid diagnosis is required [4]. Ascertainment of H.pylori infection can be made by both invasive and non-invasive methods. Invasive methods require an endoscopy with biopsyspecimen, since this allows the assessment of structural lesions and histopathology to rule out malignancy, therefore this procedure is widely practiced. Invasive methods include staining, culture, rapid urease test and PCR [5].

The non invasive tests include Urea breath test (UBT), serology, stool antigen test and stool PCR [2]. Staining and culture were first described by Warren and Marshall [6]. Staining is a rapid and inexpensive method while culture requires a great deal of expertise and time. Culture however has the advantage of yielding isolates for antibiotic sensitivity testing [5]. 


\section{Original Research Article}

The rapid urease test reduces the time and expertise required to assess infection with H.pylori [4]. Polymerase Chain Reaction (PCR) has been recently included as an important molecular method in the diagnostic armamentarium of H.pylori infection [7].

Serological analysis has the advantage of being a representative sample for the whole stomach in contrast to biopsy which represents only a small area of the stomach and may miss the patchy distribution of H.pylori. However the disadvantage is that it is not rapidly responsive to antimicrobial therapy and cannot be used to evaluate the effectiveness of therapy. Urea breath test though rapid, quantitative and rapidly responsive to treatment, involves expensive instrumentation and administration of radio isotopes [8]. Although histopathology and culture of the organism are considered the gold standard for the diagnosis of $\mathrm{H}$ pylori infection, they require an invasive procedure like endoscopy which is not comfortable in all age groups, especially in children. Hence, we require arapid, accurate and reliable noninvasive methods [9].

H.pylori antigen detection in stool is a simple rapid, non-invasive, easy to perform test that can be used to detect active infection, monitor effectiveness during therapy and to confirm cure after antibiotic use [10] The ease to collect the specimen, especially in children, in whom endoscopy would be difficult and the non requirement of specially trained staff to collect and perform the test adds to the tests advantage. The sensitivity and specificity of this test, compared to the other detection methods of H.pylori infection have been confirmed and documented [11]. This study was undertaken to evaluate the diagnostic accuracy of the rapid stool antigen test in comparison with the RUT, culture, histopathology as the gold standard methods.

\section{Materials and Methods}

Patients; 100 patients of dyspepsia were recruited in the study after undergoing upper gastrointestinal tract endoscopy. The upper GI endoscopy suggested H.pylori infection in these recruited patients. Institutional ethical clearance was obtained for this study. Informed consent was obtained from all participants. Inclusion criteria: Clinically suspected cases of dyspepsia, patients not on proton pump inhibitors for last 2 weeks and antibiotics for the last 1 month. Exclusion criteria: Patients on anticoagulants and patients having bleeding disorders. Sample collection:Two antrum and two corpus biopsy specimens were taken from each patient using a biopsy forceps through a fiberoptic gastroduodenoscope. The first specimen was inoculated into urease broth for RUT, the second specimen collected in Stuarts transport medium for culture, the third specimen collected in $10 \%$ formalin for histopathology- Giemsa stain, the last specimen was used to make an imprint smear by placing in between 2 slides and pressing gently for grams staining.

Stool Antigen test: The recruited patients were asked to collect stool sample and submit the same day or the next day to the laboratory. H.pylori Ag was detected in stool sample by SD BIOLINE H.pylori Ag rapid test procedure. This rapid test is an immunochromatographic technique. Principle: The SD BIOLINE H.pylori Ag rapid test kit result window has 2 pre-coated lines, $\mathrm{T}$ (H.pylori Ag test line) and C (control line).The test line is coated with mouse monoclonal anti Helicobacter pylori and control line, with goat anti mouse Ig gold conjugate.Both the test line and the control line are not visible prior to the test procedure. The control line is used for procedural control and should always appear if the test procedure is performed correctly.

\section{Procedure of the test}

Extraction procedure: The assay diluent and the stool sample, about $50 \mathrm{mg}$ are mixed in the sample collection tube and left to settle for $5 \mathrm{~min}$. Assemble the dropping cap on the sample collection tube for test procedure.

Test Procedure: Add3 drops (about 100 micro lit) from the sample collection tube into the sample well (S) of the test device. As the test begins to work, you will see purple color move across the result window in the center of the test device. Interpretthe test results at 10 15 minutes.

\section{Interpretation of the test}

Negative Result: The presence of only control band (C) within the result window indicates a negative result.

Positive Result: The presence of two-colorbands, test band (T) and control band (C) withinthe result window.

Invalid Result: If the control band is not visible within the result window.

Statistical analysis; The sensitivity, specificity, PPV, NPV and accuracy of the H.pylori stool antigen test was calculated and compared with the conventional standard methods (RUT, histopathology and culture).

\section{Results}

The diagnosis of H.pylori was defined as positive for culture alone or for histopathology and RUT results by gold standard methods. A positive result by H.pylori stool antigen test was taken as positive for H.pylori infection. 


\section{Original Research Article}

Of the 100 patients enrolled and tested 63 were diagnosed as positive and 37 negative for H.pylori infection by the biopsy based gold standard methods. Meanwhile,59 were positive and 41 were negative by the rapid H.pylori stool antigen test (Table-1).

Table-1: Gold Standard Tests V/s H.Pylori Stool Agent Test

\begin{tabular}{|c|c|c|c|}
\hline \multirow{2}{*}{ H. Pylori Stool Antigen Test } & \multicolumn{2}{|c|}{ Gold Standard Test } & \multirow{2}{*}{ Total } \\
\cline { 2 - 3 } & Positive & Negative & 59 \\
\hline Positive & 54 & 5 & 41 \\
\hline Negative & 9 & 32 & $\mathbf{1 0 0}$ \\
\hline Total & $\mathbf{6 3}$ & $\mathbf{3 7}$ & \\
\hline
\end{tabular}

By the gold standard tests considered (culture or RUT and histopathology positive) a total of 63 cases had H.pylori infection, 54 out of which were positive by H.pylori rapid stool antigen test, 9 cases could not be picked by the stool antigen test.This false negative result could be due tothe intermittent shedding of the H.pylori in stool. The 5 false positive which were negative by the standard tests, culture or RUT and histopathology could be due to cross reaction with other species of Helicobacter which colonize the stomach.

Table-2: Positivity of Different Laboratory Tests Employed to Detect H.Pylori in 100 cases

\begin{tabular}{|c|c|c|}
\hline Laboratory Test & No. of positive for H. Pylori & Percentage of Positivity \\
\hline Rapid urease test & 72 & $72 \%$ \\
\hline Gram's stained biopsy smear & 54 & $54 \%$ \\
\hline Histology & 63 & $63 \%$ \\
\hline Culture & 18 & $18 \%$ \\
\hline Stool antigen test & 59 & $59 \%$ \\
\hline
\end{tabular}

From the table 2 it is obvious that rapid urease test was positive in highest number of cases i.e., $72 \%$ and culture was least positive i.e., $18 \%$ of cases, this is because of the fastidious nature of H.Pylori. $63 \%$ positivity rate detected by histopathological examination by Giemsa stain. $54 \%$ positive by grams staining of the imprint smear of the gastric biopsy.

Table-3: Sensitivity of Stool Antigen Test.

\begin{tabular}{|c|c|c|c|c|c|}
\hline $\begin{array}{c}\text { TruePositive } \\
\text { (TP) }\end{array}$ & $\begin{array}{c}\text { True Negative } \\
\text { (TN) }\end{array}$ & $\begin{array}{c}\text { False Positive } \\
\text { (FP) }\end{array}$ & $\begin{array}{c}\text { False Negative } \\
\text { (FN) }\end{array}$ & Sensitivity & Specificity \\
\hline 54 & 37 & 5 & 9 & $85.7 \%$ & $88 \%$ \\
\hline
\end{tabular}

The sensitivity, specificity, PPV, NPV and overall accuracy of the HpSA test were $85.7 \%, 88 \%$, and $91.5 \%, 80.4 \%$ and 91\% respectively (Table-2).

\section{Discussion}

H.pylori colonizes the human stomach during childhood and survives in the human stomach, the only niche known to date, for the lifetime of the carrier [12]. In most of the individuals H.pylori infection may be asymptomatic. Around $20 \%$ to $30 \%$ of the infected individuals may develop peptic ulcer disease, and less than 2\% gastric cancer [13].Gastrointestinal endoscopy has been widely performed for gastrointestinal disorders and H.pylori infection diagnosis. Nonetheless, the Maastricht III consensus report recommended in primary care, a test and treat strategy using a non invasive test in adult patients with dyspepsia [14].
Invasive methods of diagnosis require endoscopy to be done, which is not suitable for primary care physicians. Hence, in the absence of endoscopic facilities the primary physicians require non-invasive methods to diagnose H.pylori infection [15]. The urea breath test, stool antigen tests and serological tests with a high accuracy are the non invasive tests which should be performed for the diagnosis of H.pylori infection [14]. Since there is evidence that infected individuals excrete $\mathrm{H}$ pylori in feaces, an immunoassay (EIA, HpSA) has been developed that can detect $\mathrm{H}$ pylori antigen in human feces. Besides, Helicobacterpylori in feces could 


\section{Original Research Article}

be detected by PCR or even culture. Therefore it is noninvasive; HpSA immunoassay would be particularly important for patients for whomendoscopy is not clinically indicated. The premier platinum HpSA test is an enzyme immunoassay (EIA) that detects anH pylori antigen present in human stools [15]. For eradication control, ideally after three months of treatment, the breath test rapidly confirms the disappearance of H.pylori, unlike the serological techniques, which need a prolonged period of time to confirm the eradication effect. Thus, because antibody titres can take up to six months to fall after successful treatment, serological tests cannot readily be used to assess the efficacy of H.pylori eradication regimens shortly after treatment [16].

Even though the radiation exposure from 14C-urea breath test is less than $1 \%$ of that received from an upper gastrointestinal series [17], and the test has $100 \%$ specificity and sensitivity [18], the long half life (5.730 years) of $14 \mathrm{C}$ restricts its use due to the environmental protection policy. That means 13C-urea breath test and the stool antigen test are the only choices of noninvasive methods for the H.pylori-infection eradication control.

The present study was therefore planned for comparative evaluation of conventional methods i.e., RUT, grams staining, histology and culture with stool antigen test for diagnosis of H.pylori infection in cases of dyspepsia. The H.pylori stool antigen test was reported in the America Gastroenterology week in 1997. The HpSA test has been widely evaluated around the world, with a weighted mean sensitivity and specificity of $90-98 \%$, respectively [ 19,20$]$. The stool antigen test may be performed by conventional immunoassay (EIA) and by rapid lateral flow immunoassay.

We tested a rapid lateral flow immunoassay stool antigen, as it is useful for small laboratories and it is faster than the conventional EIA [20]. The results of the HpSA test in comparison with the gold standard methods, obtaineda sensitivity of $85.7 \%$ and specificity of $88 \%$ which was clinically significant for the diagnosis of H.pylori infection. The results of this study were similar to various other studies evaluating the accuracy of H.pylori stool antigen test for diagnosing H.pylori infection.

$\mathrm{Li} \mathrm{YH}$ et al evaluated the clinical value of HpSA for detecting H.pylori infection. They did a similar study in 53 patients who had undergone upper endoscopy due to gastrointestinal symptoms. They evaluated the accuracy of the stool antigen test, Immuno Card STAT HpSA, against the gold standard (RUT \& Warthin- Starry staining or culture) for the diagnosis of $\mathrm{H}$. pyloriinfection. The sensitivity and specificity of the Immuno Card STAT HpSA were $92.6 \%$ and $88.5 \%$ respectively, with an accuracy of $90.6 \%$ [21]. The results of this study co-related with our study.

Demiray E et al studied the comparison of invasive methods and two different stool antigen tests, rapid STRIPHpSA and one step simple. The sensitivity and specificity of therapid STRIPHpSA was $60 \%$ and $86 \%$, where as in our study, thesensitivity and specificity was $85.7 \%$ and $88 \%$ respectively. The sensitivity of the HpSA test was higher in our study. Their study concluded that the rapid STRIPHpSA test could be used as a routine diagnostic tool for H.pylori infection [2].

In another study Silva JMK et al validated the use of the rapid lateral flow H.pylori stool antigen test as an alternative to Urea Breath Test for diagnosis of H.pylori infection in developing countries. The sensitivity and specificity of the rapid stool antigen test was $88 \%$ and $87.5 \%$ respectively.The results of their study co-related with the results of our study. Silva JMK et al considered Urea Breath Test as the gold standard, non-invasive technique for H.pylori infection diagnosis [16].

Syam et al evaluated the accuracy of HpSA for the detection of H.pylori infection in dyspeptic patients. The sensitivity and specificity of HpSA were $66.7 \%$ and $78.9 \%$ respectively. They concluded that HpSA stool test may be useful for the primary diagnosis of H.pylori infection in peptic ulcer patients [15]. Choi $\mathrm{J}$ et al conducted a prospective study, where 515 patients were enrolled in the study. They evaluated the efficacy ofa new stool antigen test for the detection of H.pylori in comparison with histology, RUT, UBT \& serology.

Sensitivity, specificity, positive and negative predictive values, and accuracy of the stool antigen test were $93.1 \%, 94.6 \%, 95.1 \%, 92.3 \%$, and $93.8 \%$, respectively. The sensitivity and specificity of the above study was slightly higher when compared to our study (sensitivity$85.7 \%$, specificity- $88 \%$ ). They concluded that the performance of a new stool antigen test was comparable to that of other methods in the diagnosis of $\mathrm{H}$. pylori infection for the screening population [22]. Pourlak T et al studied the role of HpSA test in the diagnosis ofHelicobacter Pylori infection in patients with upper GI complaints undergoing diagnostic endoscopy.

RUT and histology were used as gold standard for comparison of results. They observed that HpSA had sensitivity, specificity, PPV, NPV and accuracy of $96.67 \%, \quad 93.33 \%, \quad 93.55 \%, \quad 96.55 \%$ and $95 \%$ 


\section{Original Research Article}

respectively thus concluding the tests significant role as a diagnostic tool [23]. The results (sensitivity, specificity \& PPV-96.67\%, 93.33\%, 93.55\%) their study was significantly higher when compared to that of our study (sensitivity, specificity \& PPV - 85.7\%, 88\%, $91.5 \%)$.

Saha $\mathrm{R}$ et al studied the application of the stool antigen test to evaluate the burden of Helicobacter pylori infection in dyspepsia patients. A quantitative ELISA for HP antigen was performed on the stool samples of patients undergoing upper GI endoscopy. Samples showing a concentration of HP antigen higher than $0.05 \mathrm{micro}$ gram $/ \mathrm{ml}$ were considered as positive.

The study concluded that HP stool antigen testing to be superior to upper GI endoscopy for detecting HP infection. They recommended initial testing for $\mathrm{HP}$ stool antigen before initiating treatment and before carrying out any invasive procedure such as endoscopy [24]. Erzin et al compared two different stool antigen tests for the primary diagnosis of H.pylori infection in patients with dyspepsia. They used Femto Lab H.pylori EIA and Premier Platinum, the sensitivity and specificity of the monoclonal FemtoLabH. pylori EIA were $93 \%$ and $90 \%$ respectively, and Premier Platinum HpSA were $84 \%$ and $67 \%$ respectively. They concluded that FemtoLabH.pylori was an excellent tool for the primary diagnosis of H.pylori infection in patients with dyspepsia [25].

Islam et al assessed the performance of the HpSA for both the diagnosis of H.pylori infection and for confirming post therapy eradication. The sensitivity and specificity of HpSA were $67 \%$ and $100 \%$ respectively. There fore HpSA test was found to be a useful noninvasive diagnostic test for H.pylori infection as well as a useful method to assess the effectiveness of eradication of H.pylori infection [26].

Ito et al studied the role of HpSA test in comparison with UBT for the evaluation of the success of eradication therapy. The diagnostic accuracy of the UBT and the HpSA test was $94.3 \%$ and $97.1 \%$, respectively where the difference was not very significant. Therefore the HpSA test is a simple non invasive diagnostic tool for the evaluation of eradication therapy of H.pylori [27].

\section{Conclusion}

In conclusion, there is high prevalence of H.pylori infection and its associated gastrointestinal complications in developing countries like India. Prevention of these complications can be achieved through early diagnosis through conventional and affordable diagnostic methods. Therefore H.pylori stool test can be used as an alternative non-invasive test to breath test for diagnosis of primary infection of H.pylori.

Limitations- The limitations of this study are the sample size. The sample size studied is small, it requires to include more patients into this study to obtain more valuable results.

Addition to the existing knowledge by our study: The HpSA test can be used as a routine diagnostic tool for the diagnosis of H.pylori infection. We recommend an initial testing for Helicobacter pylori in dyspeptic patients by a simple non invasive method of stool HP antigen testing before endoscopy is done.

\section{Contribution from the Author}

Dr. Saipriya B: Data Collection, Analysis and Preparation of the Manuscript

Dr. Jagadevi: Data Collection, Analysis and preparation of Manuscript

Dr. Suresh K.: Analysis and Preparation of the Manuscript.

Dr. Prakash M G: Data collection, Analysis and Preparation of the Manuscript.

Findings: Nil; Conflict of Interest: None initiated Permission from IRB: Yes

\section{References}

1. Bindayna KM, Al Baker WA, Botta GA. Detection of Helicobacter pylori cagA gene in gastric biopsies, clinical isolates and faeces. Indian $\mathrm{J}$ Med Microbiol. 2006 Jul;24(3):195-200.

2. Demiray E, Yilmaz O, Sarkis C, et al. Comparison of invasive methods and two different stool antigen tests for diagnosis of $\mathrm{H}$ pylori infection in patients with gastric bleeding. World J Gastroenterol. 2006 Jul 14;12 (26):4206-10.

3. Allaker RP, Young KA, Hardie JM, Domizio P, Meadows NJ. Prevalence of Helicobacter pylori at oral and gastrointestinal sites in children: evidence for possible oral-to-oral transmission. J Med Microbiol 2002; 51(4) :312-317.

4. Atherton Jc, Blaser $\mathrm{Mj}$. Helicobacter pylori infections. In : Braunwold E, Fauci FC, Kasper DL, Hauser SL, Congo. DL, Jameson JL, editors Harrison's principles of internal medicine $15^{\text {th }}$ ed New York: MC Graw Hill: 2001; 960-3. 


\section{Original Research Article}

5. Versalovic J, Fox JG. Helicobacter. In ; Murray PR, Baron EJ, Pfeller MA, Fenoner FC, Yolken RH, editors. Manual of clinical microbiology. $7^{\text {th }}$ ed. Washington DC: American Society for Microbiology, 1999; 727 38.

6. Marshall BJ, Warren JR. Unidentified curved bacilli in the stomach of patients with gastritis and peptic ulceration. Lancet. 1984 Jun 16;1(8390):1311-5.

7. Mishra KK, Srivastava S, Dwivedi PP, et al. UreC PCR based diagnosis of Helicobacter pylori infection and detection of cag A gene in gastric biopsies. ndian $\mathrm{J}$ Pathol Microbiol. 2002 Jan;45(1):31-7.

8. Blaser MJ. Helicobacter pylori and related organisms. In: Mandell GL, Bennett's Principles and practice of infectious disease. $5^{\text {th }}$ ed. Philadelphia: Churchill Livingstone. 2000; 2285-93.

9. Kabir S. Detection of Helicobacter pylori in faeces by culture, PCR and enzyme immunoassay. J Med Microbiol. 2001 Dec; 50 (12): 1021-9. DOI:10.1099/ 0022-1317-50-12-1021

10. Martin JJ, Anbumani N, Kalyani M, et al. H. pylori antigen detection in stool. Indian J Med Microbiol. 2006 Jan;24(1):79-80.

11. Vaira D, Malfertheiner P, Mégraud F, et al. Noninvasive antigen-based assay for assessing Helicobacter pylori eradication: a European multicenter study. The European Helicobacter pylori HpSA Study Group. Am J Gastroenterol. 2000 Apr;95(4):925-9. DOI:10.1111/j. 1572-0241.2000.01931.x

12. Carroll IM, Ahmed N, Beesley SM, Khan AA, Ghousunnissa S, Morain CA, et al. Microevolution between paired antral and paired antrum and corpus Helicobacter pylori isolates recovered from individual patients. J Med Microbiol.2004; 1 July;53:669-77.

13. Kusters JG, van Vliet AH, Kuipers EJ. Pathogenesis of Helicobacter pylori infection. ClinMicrobiol Rev. 2006Jul;19(3):449-90.DOI:10.1128/CMR.00054-05

14. Malfertheiner P, Megraud F, O'Morain C, et al. Current concepts in the management of Helicobacter pylori infection: the Maastricht III Consensus Report. Gut. 2007 Jun;56(6):772-81. Epub 2006 Dec 14. DOI: 10. 1136/gut.2006.101634

15. Syam AF, Rani AA, Abdullah M, Manan C, Makmun D, SimadibrataM,et al. Accuracy of Helicobacter pylori stool antigen for the detection of
Helicobacter pylori infection in dyspeptic patients. World J Gastroenterol 2005; Jan 21, 11(3): 386-8.

16. Silva JM, Villares CA, Monteiro Mdo S, et al. Validation of a rapid stool antigen test for diagnosis of Helicobacter pylori infection. Rev Inst Med Trop Sao Paulo. 2010 May-Jun;52(3):125-8.

17. Marshall BJ, Plankey MW, Hoffman SR, et al. A 20-minute breath test for helicobacter pylori. Am J Gastroenterol. 1991 Apr;86(4):438-45.

18. Mattar R, Silva FM, Alexandrino AM, et al. Validation of 14C-urea breath test for diagnosis of Helicobacter pylori. Rev Inst Med Trop Sao Paulo. 1999 Jan-Feb;41(1):3-8.

19. Metz DC. Stool testing for Helicobacter pylori infection: yet another noninvasive alternative. Am J Gastroenterol. 2000 Feb;95(2):546-8.

20. Monteiro L, de Mascarel A, Sarrasqueta AM, et al. Diagnosis of Helicobacter pylori infection: noninvasive methods compared to invasive methods and evaluation of two new tests. Am J Gastroenterol. 2001 Feb;96 (2): 353-8. DOI:10.1111/j.1572-0241.2001.03518.x

21. Li YH, Guo H, Zhang PB, et al. Clinical value of Helicobacter pylori stool antigen test, Immuno Card STAT HpSA, for detecting H pylori infection. World J Gastroenterol. 2004 Mar 15;10(6):913-4.

22. Choi J, Kim CH, Kim D, et al. Prospective evaluation of a new stool antigen test for the detection of Helicobacter pylori, in comparison with histology, rapid urease test, (13)C-urea breath test, and serology. J Gastroenterol Hepatol. 2011 Jun;26(6):1053-9. doi: 10. 1111 j.1440-1746.2011.06705.x.

23. Pourlak T, Shokouhi B, Vahedi A. Role of Helicobacter Pylori stool antigen test in the diagnosis of Helicobacter Pylori infection. J Anal Res Clin Med 2017; 5(3):86-90.

24. Saha R, Roy P, Das S, Kaur N, et al. Application of a stool antigen test to evaluate the burden of Helicobacter pylori infection in dyspepsia patients. Indian J Pathol Microbiol. 2016 Jan-Mar;59(1):66-8. doi: 10.4103/0377-4929.174819.

25. Erzin Y, Altun S, Dobrucali A, et al. Comparison of two different stool antigen tests for the primary diagnosis of Helicobacter pylori infection in turkish patients with dyspepsia. Helicobacter. 2004 Dec; 9 (6): 657-62.DOI:10.1111/j.1083-4389.2004. 00280.x 


\section{Original Research Article}

26. Islam $\mathrm{S}$, Weilert $\mathrm{F}$, Babington R, et al. Stool antigen testing for the diagnosis and confirmation of eradication of Helicobacter pylori infection: a prospective blinded trial. Intern Med J. 2005 Sep; 35 (9): 526-9. DOI:10. 1111/ j.1445-5994.2005.00903.x

27. Ito M, Tanaka S, Kim S, et al. A combination of the Helicobacter pylori stool antigen test and urea breath test is useful for clinical evaluation of eradication therapy: a multicenter study. J Gastroenterol Hepatol. 2005 Aug; 20 (8): 1241-5. DOI:10.1111/j.1440-1746. 2005. 03928.x

\section{How to cite this article?}

Sai Priya B, Jagadevi, Suresh K, M.G. Prakash. Evaluation of Helicobacter pylori stool antigen test in comparison with conventional methods in detection of helicobacter pylori infection in dyspepsia patients. Trop J Path Micro 2018;4(7):505-511.doi:10.17511/jopm.2018.i07.05. 